\title{
Essais
}

Revue interdisciplinaire d'Humanités

Hors-série 3 | 2016

Usages critiques de Montaigne

\section{Montaigne : nouveaux regards des historiens}

\section{Anne-Marie Cocula}

\section{OpenEdition}

Journals

Édition électronique

URL : http://journals.openedition.org/essais/7026

DOI : 10.4000/essais. 7026

ISSN : 2276-0970

\section{Éditeur}

École doctorale Montaigne Humanités

\section{Édition imprimée}

Date de publication : 1 novembre 2016

Pagination : 146-158

ISBN : 979-10-97024-00-0

ISSN : 2417-4211

\section{Référence électronique}

Anne-Marie Cocula, "Montaigne : nouveaux regards des historiens », Essais [En ligne], Hors-série 3 | 2016, mis en ligne le 26 février 2021, consulté le 17 mars 2021. URL : http://journals.openedition.org/ essais/7026; DOI : https://doi.org/10.4000/essais.7026 


\section{Montaigne : nouveaux regards des historiens}

\section{Anne-Marie Cocula}

Rien n'est plus stimulant que la transversalité des sciences humaines au fur et à mesure de leurs avancées dans leurs différents domaines. Une preuve en est donnée par les nouvelles approches conjuguées qu'elles apportent sur Montaigne et les Essais, sans oublier ses autres écrits, découverts plus tardivement comme son Journal de voyage et sa correspondance auxquels s'ajoute la récente publication de pièces manuscrites rassemblées, transcrites et annotées avec patience et minutie par Alain Legros dans Montaigne manuscrit, un ouvrage qui porte bien son nom ${ }^{1}$. Ces avancées, du côté des historiens, peuvent se résumer par un même terme, celui de singularité, pour qualifier deux approches complémentaires : l'une, portant sur la nature des Essais, l'autre, sur le rôle politique de Montaigne comme maire de Bordeaux entre 1581 et 1585 , à la fois correspondant du maréchal de Matignon, représentant en Guyenne du roi Henri III et conseiller occasionnel du roi Henri de Navarre, futur Henri IV, devenu le premier prétendant au trône de France après la mort du duc d'Anjou, le plus jeune des fils de Henri II et Catherine de Médicis, décédé en juin 1584 lors du dernier mandat municipal de Montaigne.

La singularité littéraire des Essais, soulignée par Montaigne lui-même dans son avant-propos "Au Lecteur » et saluée par ses contemporains, se trouve confirmée par les résultats des recherches menées sur les écrits du for privé sur une longue durée allant de la fin du Moyen Age jusqu’à la première guerre mondiale. À l'exception des Essais, d'autres écrits de Montaigne, notamment l'Ephéméride et le Journal de Voyage, se trouvent concernés par cette enquête au long cours, fruit d'une heureuse collaboration entre histoire, histoire de l'art, littérature et sociologie ${ }^{2}$. Quant à la singularité de l'action politique

1 Alain Legros, Montaigne manuscrit, Paris, Classiques Garnier, 2010.

2 Ces travaux sur les écrits du for privé ont été menés dans le cadre d'une action labellisée par l'ANR, dirigée par Jean-Pierre Bardet et François-Joseph Ruggiu. Parmi les diverses publications, on retiendra les ouvrages suivants : Car c'est moy que je peins. Écritures de soi, individu et 
de Montaigne, elle résulte en grande partie de sollicitations extérieures de la part de grands personnages qui l'utilisent pour ses compétences et sa connaissance de l'histoire, passée et présente, de la province de Guyenne, auxquelles s'ajoutent son expérience d'ancien conseiller du parlement de Bordeaux qu'il a quitté en 1570 et sa renommée d'écrivain à partir de la publication des Essais, en 1580. Ce sont là des atouts précieux pour ceux qu'il côtoie ou conseille mais bien insuffisants pour lui conférer pouvoir et influence au sein d'un gouvernement monarchique dont il n'est qu'un rouage occasionnel dans une période compliquée et jalonnée d'événements imprévus, sinon imprévisibles, dont le plus choquant fut le massacre parisien de la Saint-Barthélemy en août 1572, prolongé en province par la "saison des Saint-Barthélemy" de l'automne $1572^{3}$.

\section{Singularité des Essais par rapport aux écrits du for privé}

Montaigne, lui-même, a souhaité signaler cette singularité en décidant d'abandonner la tenue des livres de raison ou livres de comptes héritée de ses ascendants, les Eyquem, anciens marchands-négociants de Bordeaux installés dans la ville au moins depuis la fin du XIV siècle. Ce choix délibéré de rupture avec ses ancêtres lui fournit l'occasion de donner une définition quasi-parfaite de ce type d'écrits et de leur raison d'être, à la fois pratique et sentimentale :

En la police oeconomique mon pere avoit cet ordre, que je scay loüer, mais nullement ensuivre. C'est qu'outre le registre des negoces du mesnage, où se logent les menus comptes, payements, marchés, qui ne requierent la main du Notaire, lequel registre, un Receveur a en charge : il ordonnoit à celuy de ses gents, qui luy servoit à escrire, un papier journal, à inserer toutes les survenances de quelque remarque, et jour par jour les memoires de l'histoire de sa maison : tres-plaisante à veoir, quand le temps commence à en effacer la souvenance, et tres à propos pour nous oster souvent de peine : Quand fut entamee telle besoigne, quand achevee : quels trains y ont passé, combien arresté : noz absences, mariages, morts : la reception des heureuses et malencontreuses nouvelles : changement des serviteurs principaux : telles matieres. Usage ancien, que je trouve bon à rafraichir, chacun en sa chacuniere : et me trouve un sot d'y avoir failli. (I, 34, 229-230) ${ }^{4}$

liens sociaux (Europe, $X V^{E}-X X^{e}$ siècle), éd. Sylvie Mouysset, Jean-Pierre Bardet et François-Joseph Ruggiu, Toulouse, Méridiennes, 2010 ; Écritures de familles, écritures de soi (France-Italie, XVI'XIXe siècles), éd. Michel Cassan, Limoges, Pulim, 2011 ; L’Amitié dans les écrits du for privé et les correspondances de la fin du Moyen Age à 1914, éd. Maurice Daumas, Pau, PUPPA, 2014.

3 Denis Crouzet, La Nuit de la Saint-Barthélemy. Un rêve perdu de la Renaissance, Paris, Fayard, 1994.

4 Montaigne, Les Essais, éd. Jean Balsamo, Michel Magnien et Catherine Magnien-Simonin, Paris, Gallimard, 2007, I, 34, p. 229-230. Nous citerons désormais dans le texte les Essais d'après cette édition de référence. 
Tout est précisé dans cette description en forme d'autocritique puisqu'elle apparaît dans un ajout postérieur à l'édition des Essais de 1588. Montaigne sait qu'il n'a ni le temps, ni le loisir de revenir en arrière pour réparer ce qui a été perdu par sa faute. Seul persiste le regret. À la place de la tenue régulière d'un livre-journal, il s'est efforcé, tant bien que mal, d'alimenter en nouvelles son agenda, le Beuther, acquis dans sa jeunesse pour y noter les principaux événements de son existence et de celles de ses proches.

La transcription et l'analyse de cette Ephemeris historica ont suscité une biographie particulière dont l'initiateur fut, en 1855, le docteur JeanFrançois Payen et, le dernier en date, Legros, dans son ouvrage Montaigne manuscrit, avec un soin porté à la langue, à ses variations dans le temps, à la graphie des mots et à la couleur des encres ${ }^{5}$. À tout prendre - Legros a raison de le souligner - cet éphéméride ne fait pas l'objet d'une attention régulière de la part de Montaigne comme en témoignent ses lacunes chronologiques, ses retours en arrière, ses ratures et ses suppressions. En fait, il remplit l'office d'un livret de famille à l'intention de ses proches et de sa descendance avec la mention des naissances ou baptêmes de ses filles dont une seule, Léonor, née le 9 septembre 1571, survivra. Pour les autres, mortes quelques jours ou quelques mois après leur naissance, les dates de leurs décès figurent à la suite de celles de leurs baptêmes et des noms de leurs parrains et marraines. Seulement trois d'entre elles sont désignées par leurs prénoms : Thoinette, née en 1570, "le premier enfant de mon mariage ", Anne, née le 5 juillet 1573, Marie, la dernière, née le 21 février 1583.

Si quelques lignes suffisent à Montaigne pour inscrire sur le Beuther les mentions d'actes familiaux, il n'en est plus de même des récits historiques qui le concernent directement. Le plus circonstancié et le plus précis décrit, à la date du 19 décembre 1584, la venue du roi Henri III de Navarre chez lui, dans sa demeure périgourdine, en compagnie de grands personnages de son entourage, cités dans un ordre de préséance qui n'a pas échappé à Montaigne et qui succède au relevé méticuleux des signes de confiance du roi de Navarre à son égard : deux jours passés ensemble avec un service assuré par les seuls serviteurs de Montaigne sans intervention des " officiers " du prince, sans test de nourriture par crainte de poison et avec un repos dans le lit du maître de céans selon les règles bien établies d'une hospitalité seigneuriale.

Confiance d'autant plus précieuse et digne d'être signalée qu'au même moment, à Paris et en Lorraine, est conclue l'alliance entre Français et Espagnols qui scellent, dès janvier 1585, la naissance de la sainte Ligue

5 Alain Legros, Montaigne manuscrit, op. cit., p. 69-102. 
catholique avec l'objectif d'empêcher par tous les moyens l'accession sur le trône de France d'un prétendant protestant. Telle est, en effet, la situation d'Henri de Navarre à la suite du décès, en juin 1584, du duc d'Anjou, le dernier frère du roi Henri III, lui-même sans héritier. Montaigne assume jusqu'au bout son rôle de maître de céans en offrant au roi de Navarre sa distraction favorite : deux jours à courir le cerf dans sa forêt. L'accomplissement de sa mission, a fortiori bien délicate, a droit dans l'éphéméride à un traitement de faveur souligné par Alain Legros : "Ecriture et mise en page soignées, à la mesure de l'événement : ductus régulier, encre sombre, absence de ratures, utilisation de tout l'espace disponible grâce à une bonne mise en page ${ }^{6}$.

Une considération semblable entoure le récit fait par Montaigne de son emprisonnement à la Bastille, le 10 Juillet 1588, dans Paris, la capitale ligueuse aux mains des partisans des Guise. Henri III a dû la quitter précipitamment, le 13 mai, pour échapper à l'encerclement des barricades qui menacent sa résidence du Louvre. Montaigne s'y prend à deux fois pour conter sa mésaventure. Une première version, datée du 20 juillet, est systématiquement barrée par ses soins avec une encre plus sombre qui lui a servi pour la nouvelle rédaction de son récit avec des variantes significatives. Si la raison de son emprisonnement reste inchangée puisqu'il a servi d'otage en échange de l'emprisonnement à Rouen d'un gentilhomme ligueur, cette version met davantage en valeur la mobilisation de grands personnages, pour lors adversaires, mobilisés en sa faveur pour le faire sortir promptement de prison. Parmi eux figurent les deux grands acteurs de la scène parisienne : la reine mère Catherine de Médicis et le duc Henri de Guise, alors en négociations secrètes pour éviter le pire de la part des ligueurs extrémistes, sans oublier le rôle-clé du ministre Villeroy, médiateur entre le roi et les partis en présence, notamment les ligueurs.

Juste après ces deux récits qui n'en font plus qu'un, la note suivante du Beuther est d'un laconisme surprenant qui traduit, à mots à peine couverts, l'importance de la nouvelle et la gravité de ses conséquences : " 1588, Henri, duc de Guise, à la vérité des premiers hommes de son âge, fut tué en la chambre du roi» (p. 98). Il ne souhaite pas en écrire davantage sur les exécutions du duc Henri de Guise et de son frère, le cardinal de Lorraine, tous deux exécutés au château de Blois sur ordre de Henri III. C'est à Blois où se tiennent les États généraux que Montaigne s'était rendu en octobre 1588, à l'occasion de leur ouverture. Désormais, il est de retour chez lui depuis novembre, donc tout excusé de ne pas développer davantage après avoir dit l'essentiel : son admiration pour Henri de Guise et l'endroit exact de sa mise à mort, la chambre du roi. 
Par contre, si l'on remonte le temps, l'éphéméride passe sous silence le séjour du roi de Navarre chez Montaigne dans la nuit du 23 au 24 octobre 1587. Quelques jours après la victoire quasi-miraculeuse qu'il vient de remporter à Coutras, le 20 octobre, contre l'armée royale du duc de Joyeuse. Le favori du roi, qui avait reçu mission de s'emparer du "Béarnais » comme l'appelaient les ligueurs, fut tué au cours du combat en même temps qu'un de ses jeunes frères ${ }^{7}$. Pourquoi ce silence de la part de Montaigne et de la plupart des sources puisque la seule mention de cette courte halte de Henri de Navarre chez Montaigne se trouve dans le registre comptable de ses dépenses au château de $\mathrm{Pau}^{8}$. Est-ce par crainte d'un avenir incertain du prétendant au trône de France ou par impossibilité de juger du retentissement de son exploit militaire? Car, quelques jours plus tard, Henri de Guise rééquilibre leurs chances respectives en remportant deux victoires sur les «reitres », ces mercenaires allemands venus en renfort pour aider les protestants. La première victoire de Guise a lieu à Vimory, près de Montargis, le 26 octobre, et atténue à la Cour et dans Paris la mémoire douloureuse de Coutras. La seconde se déroule à Auneau, le 24 novembre. Il revient au duc d'Epernon, l'archi-favori d'Henri III, débarrassé de son rival, le duc de Joyeuse, de négocier le départ des mercenaires hors de France.

Le sort du royaume restait en suspens même si la victoire de Coutras avait assuré le salut du roi de Navarre. Mais pour combien de temps si ses adversaires, royaux et ligueurs, restaient unis dans leur détermination à l'empêcher de briguer le trône de France ? Peut-être convient-il d'envisager comme le fait Michel Cassan à propos du Journal d'Elie de Roffignac, un noble corrézien contemporain de cette période, que les "écritures de soi " contiennent des chapitres volontairement absents ou transcrits de manière laconique pour préserver l'existence de leur auteur et l'avenir des siens. De la sorte, tout diagnostic politique s'avère aléatoire : "il est impossible de déterminer, preuves à l'appui, les inclinations d'Elie de Roffignac et de décider s'il est un ligueur, un crypto-ligueur ou un catholique royaliste resté fidèle à son souverain ${ }^{9}$ ".

Aussi toute analyse de l'éphéméride, en sa qualité d'écrit du for privé, doit être rattachée aux circonstances de la rédaction de ses notes ellesmêmes dépendantes des événements que Montaigne souhaite inscrire ou non dans une mémoire familiale de trois générations. Celle qui l'a précédé avec pour principal représentant son père, Pierre Eyquem, décédé en

7 Nicolas Le Roux, La faveur du roi, Mignons et courtisans au temps des derniers Valois (vers 1547vers 1589), Seyssel, Champ Vallon, 2000, p. 494, p. 614-619.

8 Jean-Claude Cuignet, Litinéraire d'Henri IV, Les 20597 jours de sa vie, Pau, Héraclés, Société Henri IV, 1997, p. 85.

9 Michel Cassan, «Le journal d'Elie de Roffignac ou l'absence de l'écriture de soi ? ", in Écritures de famille, écritures de soi, op. cit., p. 157-172. 
juin 1568 ; celle qui lui est contemporaine en sa qualité de frère aîné à la tête d'une nombreuse fratrie pour le moins difficile à contenir ; enfin, celle de ses descendants, aussi ténue soit-elle, avec pour relais sa fille Léonor, puis sa petite-fille Françoise de La Tour, née un an avant sa mort. C'est à leur intention qu'il fait entrer dans l'histoire familiale les deux épisodes du 19 décembre 1584 et du 10 juillet 1588. Non seulement, ils rehaussent sa personne et son rôle à des moments importants de l'histoire, mais encore, sans son témoignage, ils n'auraient laissé aucune trace historique tant ils semblent anecdotiques et peu dignes d'intérêt aux yeux des personnages de premier plan qu'il a côtoyés.

En même temps, ils illustrent la nature et le niveau des services qu'il a rendus et qui s'apparentent à ceux d'un Elie de Roffignac, dont la renommée " locale " lui vaut d'être consulté par " des hommes de pouvoir " quel que soit leur parti : "On le voit, Roffignac réussit à remplir un rôle de médiateur et de négociateur auprès de nobles cultivant des engagements confessionnels et des fidélités politiques contrastées et antagonistes ${ }^{10}$ ". À force de missions délicates accomplies sur le terrain, le même Elie de Roffignac a été invité aux trois assemblées primaires et concurrentes chargées de désigner les députés du Bas-Limousin aux Etats de Blois, en 1588. Il n'aura pas l'occasion d'être élu et d'y rencontrer Montaigne qui termine ainsi l'année tourmentée passée à Paris puis en Picardie chez Marie de Gournay. Mais le principal résultat le plus tangible de ces mois difficiles et le plus prometteur reste la publication de la cinquième édition des Essais parue en juin chez Abel L'Angelier, enrichie d'un troisième livre et de plus de cinq cents additions par rapport aux deux premiers livres publiés à Bordeaux, au printemps 1580, chez Simon Millanges.

De retour chez lui, en novembre 1588, il pouvait s'estimer satisfait et sans doute heureux de recevoir quelques temps après le manuscrit de Marie de Gournay intitulé le Proumenoir de M. de Montaigne. Les corrections et les ajouts déjà portés sur l'un des exemplaires de la récente édition de 1588 lui importaient plus que tout le reste maintenant qu'il était en retrait d'une vie publique et d'un rôle de messager-médiateur qui fut le sien et dont il confesse la modestie dans un des chapitres du livre II, au titre éloquent : "A demain les affaires":

Je n'en ouvris jamais (des lettres), non seulement de celles, qu'on m'eust commises : mais de celles mesmes que la fortune m'eust faict passer par les mains. Et fais conscience si mes yeux desrobent par mesgarde, quelque cognoissance des lettres d'importance qu'il lit, quand je suis à costé d'un grand. Jamais homme ne s'enquit moins, et ne fureta moins ès affaires d'autruy. (II, 4, 382-383)

10 Ibid., p. 166-167. 
On peut la croire ou pas, mais cette confidence, née d'un commentaire suscité par sa lecture familière de Plutarque, tient dans la précision particulièrement éclairante qu'il nous révèle de sa position et de son rôle dans la société : «[...] quand je suis à costé d'un grand». De quelle catégorie de " grand " s'agit-il ? Un grand seigneur, assurément, et peut-être même un grand personnage car il en a côtoyé beaucoup. En tout cas, il s'agit d'une personne à laquelle il ne saurait se comparer et dont il se sent inférieur selon les règles d'une hiérarchie sociale qu'il ne lui est pas permis de transgresser, sauf en écriture et avec une jubilation certaine qu'il exprime à la fin du dernier chapitre des Essais, au moment d'un ultime bilan :

Nous cherchons d'autres conditions, pour n'entendre l'usage des nostres : et sortons hors de nous, pour ne sçavoir quel il y faict. Si avons-nous beau monter sur des eschasses, car sur des eschasses encores faut-il marcher de nos jambes. Et au plus eslevé throne du monde, si ne sommes nous assis, que sus nostre cul. (III, 13, 1166)

Cette proximité qu'il vit auprès des grands n'est pas différente de celle que l'on réserve à un messager auquel on confie des missives et qui se garde bien de les lire ou prétend de ne pas l'avoir fait, quitte à détourner ses regards. Enfin, détail révélateur de la position subalterne de Montaigne, ce "grand ", à côté de lui, plongé dans la lecture du courrier qu'il vient de recevoir, n'a nulle obligation de lui prêter attention et de s'adresser à lui sinon en lui confiant un message de réponse, écrite ou orale. Cette confidence du chapitre $4 \mathrm{du}$ livre II ("A demain les affaires »), trop rarement signalée, doit être mise en exergue avant toute analyse de son action publique.

\section{«Quand je suis à costé d'un grand »}

Cette analyse résulte du renouvellement considérable, depuis au moins une trentaine d'années, de l'historiographie de la période des guerres de religion, en tous domaines. Sans pouvoir citer tous les ouvrages, saluons les travaux majeurs d'historiens qui ont creusé les sillons des recherches à venir et élargi considérablement les perspectives de travaux universitaires en cours en France et à l'étranger. Parmi eux, Jean Delumeau, Jean-Pierre Babelon, Bernard Barbiche, Elie Barnavi, Philip Benedict, Yves-Marie Bercé, Jacqueline Boucher, Janine Garrisson, Myriam Yardeni, Denis Crouzet, Robert Descimon, Arlette Jouanna, Jean-Marie Constant, Mark Greengrass, Nicolas Le Roux, Emmanuel Le Roy Ladurie, Roland Mousnier, Denis Richet, Robert Sauzet, Laurent Bourquin, et bien d'autres encore, ont participé à ce renouvellement qui mériterait de faire l'objet d'une synthèse pour en mesurer le retentissement et les bouleversements au regard des historiens des générations précédentes qui n’ont pas osé ou 
voulu remettre en doute les certitudes de leurs grands ancêtres, les historiens du XIX ${ }^{e}$ siècle. Un seul exemple, biographique, peut illustrer ce bouleversement historiographique : celui de la reine Catherine de Médicis, sortie désormais de l'enfer des pamphlets protestants dont le plus fameux rédigé et diffusé en 1574 peu après les massacres de la Saint-Barthélemy avait inspiré la plupart de ses biographes : Discours merveilleux de la vie, actions et deportemens de la reyne Catherine de Medicis, declarant tous les moyens qu'elle a tenus pour usurper le gouvernement du royaume de France et ruiner l'etat d'iceluy ${ }^{11}$.

Ces travaux se sont accompagnés de la publication de sources et documents qui, sans parvenir à l'exhaustivité des publications du XIX ${ }^{\mathrm{e}}$ siècle pour des raisons de coût éditorial, fournissent des éléments indispensables à la compréhension d'une époque et d'une politique : ainsi des lettres d'Henri III, patiemment assemblées, résumées et publiées par les soins de Jacqueline Boucher ${ }^{12}$. En Guyenne, province familière à Montaigne, le contenu, la densité, la continuité et la précision des informations fournies par la correspondance du roi avec le maréchal de Matignon, son représentant dans la province depuis 1581, apportent une appréciation nouvelle des événements contemporains de l'engagement de Montaigne dans la décennie 1580-1590, celle de tous les bouleversements liés au déclenchement et au déroulement des guerres de La Ligue, considérées comme la conséquence politique des guerres de religion et l'engrenage d'une lutte pour le trône de France entre Valois, Bourbons et Guise.

Pareil constat risque de désappointer celles et ceux qui ont eu à cœur de faire de Montaigne, conseiller du parlement de Guyenne jusqu'en 1570, puis maire de Bordeaux, de 1581 à 1585, un acteur de premier plan dont l'influence et les missions entourées de discrétion auraient permis de franchir le cap tempétueux du changement de lignage dynastique, en août 1589, entre Henri III, le dernier des rois Valois, et Henri IV, le premier des rois Bourbons. Bouleversement extraordinaire dans la succession monarchique que l'avènement d'un roi protestant à l'un des pires moments de l'histoire de la royauté française. Car Henri III, son prédécesseur, fut terriblement malmené et critiqué, en proie à la vindicte de la sainte Ligue dressée contre lui depuis janvier 1585 grâce à l'alliance des Guise et du roi d'Espagne, Philippe II, et ardemment soutenue par le peuple de Paris entré en révolution en mai 1588. Obligé de fuir sa capitale, puis contraint de convoquer les états généraux à Blois, Henri III fait exécuter Henri de Guise et son frère, le cardinal de Lorraine, à la Noël 1588. Six mois plus tard, le $1^{\text {er }}$ août 1589 , il est mortel-

11 Denis Crouzet, Le haut cour de Catherine de Médicis, Paris, Albin Michel, 2005, p. 606.

12 Lettres de Henri III, roi de France, recueillies par Pierre Champion et Michel François, éd. Jacqueline Boucher, Paris, au siège de la Société ; les tomes V, VI et VII (1580-1587), publiés entre 2005 et 2012, sont une référence essentielle pour l'étude du mandat de maire de Montaigne à Bordeaux, entre 1581 et 1585 . 
lement blessé par le moine ligueur Jacques Clément. Avant de succomber, il a eu le temps de renouveler, auprès de ses proches, la désignation d'Henri III de Navarre comme son successeur légitime sur le trône de France. Les pires difficultés, politiques, militaires et religieuses attendent désormais Henri IV.

Les deux mandats successifs de Montaigne en qualité de maire de Bordeaux n'auraient pas autant attiré l'attention s'ils n'avaient été précédés de la publication, en 1580, des deux premiers livres des Essais dont un exemplaire fut présenté par leur auteur à Henri III, en juillet 1580, avant son départ pour son long voyage en Allemagne, en Suisse et en Italie. Juste avant de se rendre en Lorraine, Montaigne s'est attardé au siège de la ville de la Fère mené par le maréchal de Matignon contre les troupes protestantes du prince de Condé qui s'est emparé de la ville par surprise et par volonté farouche de revanche pour n'avoir pu prendre possession effective de son gouvernement de Picardie alors que son cousin germain, Henri de Navarre, occupe une fonction semblable en Guyenne. Nul doute que la blessure mortelle reçue pendant le siège par Philibert de Gramont, proche du roi Henri III et " ami » de Montaigne, dut lui permettre d'approcher Matignon, facilitant ainsi leurs relations ultérieures.

Son choix comme maire de la ville de Bordeaux, en août 1581, s'apparente à ceux des maires de villes soumises comme Bordeaux à un contrôle monarchique accru à la suite de révoltes durement réprimées sous les règnes de François $1^{\text {er }}$ et de Henri II avec pour mémoire vivante la dauphine devenue la reine Catherine de Médicis, puis la reine-mère des derniers rois Valois. Elle est la principale instigatrice du choix de Montaigne pour faciliter en Guyenne l'installation de son protégé, le maréchal de Matignon. Agréée en haut lieu par Henri III et Henri Navarre qui a tout intérêt à ménager Bordeaux, sa capitale, qui refuse de le recevoir depuis qu'il s'est reconverti au protestantisme en 1576, cette élection municipale au sein d'une jurade réduite à six représentants a pour but d'assurer un intérim entre deux lieutenants généraux, représentants du roi dans la province : le maréchal de Biron et le maréchal de Matignon.

Le premier qui cumulait les fonctions de maire de Bordeaux et de lieutenant général, étant devenu insupportable au roi de Navarre qui a négocié âprement son renvoi, avec la bénédiction de son épouse, Marguerite de Valois, lors des accords du Fleix conclus avec Catherine de Médicis en novembre 1580. Quant au second, issu de la clientèle de Catherine de Médicis, il encourt le reproche d'être un Normand, "étranger " en Guyenne. Lui aussi, à l'égal de Biron, doit cumuler les fonctions de maire de Bordeaux et de lieutenant général du roi, une fois mises en place les dispositions des accords du Fleix, en particulier l'installation d'une chambre mi-partie pour la conduite des procès impliquant des protestants. Des courtisans, avides de rumeurs, ont mesuré, à la manière du mémorialiste Brantôme, l'ampleur des difficultés qui 
attendent ce "froid Normand au pays des cervelles chaudes " que sont les Gascons ${ }^{13}$. Montaigne présente enfin l'avantage de ne pouvoir refuser cette désignation-élection, quels que soient son étonnement de l'apprendre et son souhait de la refuser ${ }^{14}$. Enfin, avantage de taille pour les responsables de son choix, ils savent qu'ils ne seront pas obligés, le moment venu, de lui trouver de nouvelles fonctions plus prestigieuses tant il a hâte de se remettre à son ouvrage : la suite des deux premiers livres des Essais.

Dès lors, Montaigne en a bien conscience, cet intérim ne l'oblige pas à une mobilisation de tous les instants tant à Bordeaux, qu'en Périgord où sa demeure fait office de lieu de surveillance et de rendez-vous sur le principal itinéraire des protestants entre La Rochelle et Montauban. Sa tâche est de faciliter l'adaptation bordelaise de Matignon et de poursuivre des contacts avec Henri de Navarre, désormais conseillé au plus près par Duplessis-Mornay qui, à partir de 1582, se voue avec ardeur et obstination à transformer ce prince aventurier en homme d'Etat, capable un jour prochain de monter sur le trône ${ }^{15}$. A moins d'un événement imprévu, son mandat doit être unique et réduit aux deux années réglementaires, comme celui de son père, Pierre Eyquem, entre 1554 et 1556 . Or, cet imprévu bouscule les prévisions et prend la forme d'un renforcement du parti ligueur dans Bordeaux, hostile à Montaigne et à Matignon, contraints de faire front sans cesser de contrarier les initiatives d'Henri de Navarre de plus en plus audacieux dans la pratique de coups de main et autres prises de villes par surprise. Dans Bordeaux, cette menace vise les échanges commerciaux avec pour cibles privilégiées ses avantports de Blaye et de Bourg, objets d'entreprises militaires des ligueurs et de leurs alliés espagnols.

Le second mandat de Montaigne est donc rendu nécessaire par une obligation de mise en défense généralisée de la province. Elle mobilise entièrement le maréchal de Matignon, sollicité sans arrêt par Henri III pour éviter la confrontation de deux fronts qui menacent l'intégrité de la Guyenne : le front ligueur et ses alliés espagnols, et le front protestant du roi de Navarre qui a évincé son cousin Condé auprès de leurs coreligionnaires. Période cruciale où Philippe Duplessis-Mornay assume avec ténacité son rôle de conseiller afin d'éviter une complète rupture entre Henri III et Henri de Navarre. Plus que jamais, Montaigne assume ses fonctions de messager-médiateur laissant à Matignon la tâche difficile d'assurer sa propre réélection comme maire. Cet épisode électoral qu’il n’a pas maitrisé n’est pas pour lui déplaire puisqu’il

13 Anne-Marie Cocula, Brantôme, Amour et gloire au temps des Valois, Paris, Albin Michel, 1986, p. 376.

14 Montaigne, Journal de voyage, éd. F. Garavini, Paris, Gallimard, 1983, p. 336.

15 Hugues Daussy, Les huguenots et le roi. Le combat politique de Philippe Duplessis-Mornay (15721600), Genève, Droz, 2002, p. 190. 
marque une victoire sur leurs adversaires ligueurs. Mais une victoire sans gloire personnelle qui laisse dans son éphéméride, une trace atténuée, inscrite a posteriori avec une encre plus sombre après l'annonce de sa première élection : "et lan 1583 fus continué ${ }^{16}$ ".

À cette date, en Guyenne, le principal danger est celui de la Ligue qui, bien renseignée, se prépare à anticiper la disparition du dernier des princes Valois, le duc d'Anjou, doublement affecté par son échec dans les Flandres et par les fréquents assauts de la maladie qui a déjà provoqué la mort ses frères, les rois François II et Charles IX. L'aventure malheureuse de Marguerite de Valois, exclue de la Cour par son frère Henri III et renvoyée en Guyenne où elle avance peu à peu selon les consignes dictées à distance par son époux Henri de Navarre, ajoute aux ennuis de Montaigne dans Bordeaux où grandit l'angoisse vitale du ravitaillement.

La fin légale de son mandat, en août 1585 , n'est pas obscurcie par la seule crainte de la peste qui aurait dicté sa décision de ne pas revenir à Bordeaux pour y transmettre le flambeau de maire au maréchal de Matignon, mais par la conclusion du traité de Nemours entre Henri III et la Ligue, le 18 juillet 1585. L'édit qui en résulte proclame la déchéance du roi de Navarre et oblige les protestants à l'abjuration ou à l'exil. Il a pour conséquence immédiate une vague massive de conversions au catholicisme au point de réduire de moitié en quelques années le nombre des protestants par rapport à celui de 1560 .

À quel moment Montaigne a-t-il appris cette nouvelle ? Henri III en a averti ses représentants en province dès le 19 ou le 20 juillet. Le 27, il écrit une lettre, portée par différents messagers de confiance, au vicomte de Gourdon, protestant convaincu, dont l'influence sur le roi de Navarre est bien connue, avec la consigne de le persuader de revenir au catholicisme. En échange, son passé de fidèle inconditionnel de Jeanne d'Albret sera passé sous silence. Etonnant marchandage qui se solde par un échec cinglant pour le roi puisque, le 12 août, Antoine de Gourdon adresse depuis Bergerac, la ville refuge d'Henri de Navarre, une lettre de huit pages qui met les choses au point en attaquant l'évêque de Rome (le pape), l'édit de 1585 (Nemours), l'éviction du roi de Navarre et les intrigues des princes de Lorraine. Tout incite à penser que Montaigne a eu connaissance de l'édit de Nemours avant la fin juillet puisque Henri III avait recommandé dans sa lettre à Gourdon de tenir informé de sa réponse, le plus tôt possible, le maréchal de Matignon ${ }^{17}$.

16 Alain Legros, Montaigne manuscrit, op. cit., p. 91.

17 Lettres de Henri III, roi de France, éd. Jacqueline Boucher, t. VII, mars 1585-décembre 1587, Paris, 2012, p. 112. 


\section{Conclusion}

Même si Montaigne n’en a pas terminé avec les événements politiques de son temps, force est de constater que son rôle désormais sera moins dépendant des ordres reçus de la part de Matignon et des recommandations épistolaires prodiguées par Duplessis-Mornay. Ainsi s'explique la liberté de ton contenue dans les lettres qu'il adresse à Henri IV en proie aux tourments politiques, militaires et religieux qui jalonnent sa conquête du trône avec pour épisode tragique et douloureux le siège de Paris et les terribles souffrances des Parisiens.

Depuis son départ du parlement, Montaigne est resté fidèle à ses fonctions de magistrat à l'exemple de ses anciens collègues envoyés en mission sur le terrain pour l'application des édits de pacification et la recherche de solutions d'apaisement. Son ami Etienne de La Boétie avait été très bon en ce domaine pour apaiser, aux côtés de Burie, les troubles religieux de l'Agenais et légiférer sur les conditions locales d'un édit de paix dont les clauses annoncent l'édit de Janvier 1562. Promu gentilhomme de la chambre du roi Charles IX en 1571, puis de la chambre du roi de Navarre, en 1577, Montaigne n'était pas en porte à faux comme on l'a souvent prétendu. Il suffit de lire l'abondante correspondance du roi Henri III pour y trouver les indices répétitifs de sa recherche d'une médiation religieuse et politique en faveur du roi de Navarre à mesure que disparait son espoir de la naissance d'un héritier.

Dès lors le séjour du futur Henri IV chez Montaigne à la Noël 1584 n'a rien de subversif. Il confirme à la fois la situation stratégique de la seigneurie de Montaigne sur le parcours des troupes protestantes et il anticipe sur la naissance de la sainte Ligue, en janvier 1585, que Henri III n’a pas réussi à endiguer comme il avait su le faire en 1576, en distribuant dans le royaume des formulaires d'adhésion qui détournaient vers lui les partisans de la Ligue. Après la mort du duc d'Anjou, en juin 1584, les Guise ne sont pas prêts à se laisser prendre à ce piège et choisissent de négocier avec le roi d'Espagne. Il en est finalement de même, en octobre 1587, lorsque le roi de Navarre fait étape à Montaigne à l'issue de sa victoire inespérée remportée à Coutras. Certes les relations entre Henri III et le roi de Navarre se sont envenimées jusqu’à devenir guerrières mais, là encore, la lecture des lettres du roi de France révèle son souhait, presque désespéré, de garder en vie celui qu'il considère encore comme son successeur en dépit de l'édit de Nemours de juillet 1585. Tel n'était pas le dessein des ligueurs les plus ardents de l'armée du duc de Joyeuse qui avaient juré la perte du Béarnais et des protestants. Une nouvelle fois, comme à la Noël 1584, Montaigne avait joué le rôle qu'on pouvait attendre de lui : celui d'un serviteur d'une légitimité monarchique, bien au-dessus des partis.

Anne-Marie Cocula

Université Bordeaux Montaigne 


\title{
Résumé
}

Le profond renouvellement de l'historiographie des guerres de religion en France dans la seconde moitié du XVI ${ }^{\mathrm{e}}$ siècle ainsi que la multiplication des études consacrées aux écrits du for privé permettent d'aborder différemment le rôle politique de Montaigne. De 1581 à 1585 intervient son mandat de maire de Bordeaux. Cette fonction municipale doit être reconsidérée comme un intérim de courte durée entre les fonctions de maires de Bordeaux et de lieutenants généraux du roi exercées avant lui par le maréchal de Biron et, après lui, par le maréchal de Matignon. Cette parenthèse municipale aura des prolongements imprévus dont le retentissement se lit dans l'éphéméride de Montaigne, à condition de prêter grande attention à la complexité des événements politiques et religieux et à leur chronologie.

\section{Mots-clés}

For privé, jurade, parlement, intérim, protestants, catholiques, ligueurs.

\begin{abstract}
Recent interpretations in the historiography of the Wars of Religion and the multiplication of studies related to the inwardness investigations of the author enable us to approach the political role of Montaigne differently. From 1581 to 1585 , that is to say during his time as mayor of Bordeaux, Montaigne's public function must be seen as a short interim, somewhat different from the responsibilities of the previous and following mayors and marshals of Guyenne such as Biron and Matignon. This transition will nonetheless have unforeseen repercussions, as can be witnesses in Montaigne's Book of Reason (éphéméride), if we pay close attention to the complexity of political and religious events and their chronology in this document.
\end{abstract}

\section{Keywords}

Inwardness, jurade, Parlement, interim office, Protestants, Catholics, Catholic Ligue. 